\title{
Duodenal hematoma secondary to acute chronic pancreatitis: case report and literature review
}

\begin{abstract}
Introduction: Duodenal intramural hematoma is a rare consequence of pancreatitis, which occurs with obstruction of the duodenum, and may evolve with rupture of the organ.

Case report: 47 year-old male presenting abdominal pain and several episodes of vomiting. The upper gastrointestinal endoscopy and abdominal tomography confirmed the presence of duodenal intramural hematoma and pancreatitis. Conservative treatment was chosen, presenting a satisfactory response. However, it evolved with rupture of the hematoma and death
\end{abstract}

Discussion and Conclusion: duodenal intramural hematoma caused by pancreatitis is poorly reported in the literature, its treatment may be conservative or surgical, consideration should be given to the patient's clinical status and degree of duodenal involvement.

Keywords: pancreatitis, duodenal hematoma, intramural hematoma
Volume 9 Issue I - 2019

Luiz Carlos de Araújo Souza,' Rafael

Francisco Alves Silva,' Carlos Hirokatsu

Watanabe Silva, ${ }^{2}$ Oliver Rojas Claros $^{3}$

'Undergraduates of Medicine in the University Center of

Brasilia, Brazil

${ }^{2}$ Physician Urologist, Department of Uro-Oncology of Hospital Santa Lúcia, Brazil

${ }^{3}$ Physician Surgeon of the Hospital Israelita Albert Einstein, Brazil

Correspondence: Luiz Carlos Araújo Souza, Researcher of the Department of Cytopathology and Pathological Anatomy of the Base Institute of the Federal District (NUCAP-IHBDF), St. Hospital Medical South-Asa Sul, Brasilia, Brazil,

Email luiz_carlos5@hotmail.com

Received: February 10, 2019 | Published: February 19, 2019

\section{Introduction}

Intramural duodenal hematoma (HID) was first reported in 1838 by Mc Lauchlan. ${ }^{1}$ Blunt abdominal trauma accounts for $70 \%$ of cases, other causes are related to endoscopic procedures and coagulopathies..$^{2-4}$ There are rare cases of HID associated with acute and chronic pancreatitis, the pathophysiology of this association is uncertain. The treatment of HID is controversial, and may be conservative or surgical. ${ }^{2}$ Aim of this study is to report a case of HID secondary to pancreatitis and review the most appropriate treatment in the literature.

\section{Case report}

A47-year-old male was admitted to the emergency surgical service with a history of severe epigastric pain associated with sweating that started one week after admission. He reported that the symptoms were triggered by the ingestion of distillates with spontaneous improvement. One day after hospitalization, he presented an episode of intense epigastric pain associated with lipothymia, nausea and multiple episodes of vomiting after feeding. It refers to similar recurring episodes of pain for 11years, with increased frequency in the last year. The patient reported daily habit for 20years ( $150 \mathrm{~g} /$ day). $\mathrm{He}$ had no other comorbidities. At the entrance examination, he was in a regular general condition, dehydrated, nor motensive, tachycardic (105bpm), afebrile, eupneic. It presented pain on palpation diffusely, worse on right and epigastrium hypochondrium.

Abdominal ultrasonography demonstrating a heterogeneous expansive lesion with imprecise limits located laterally to these and portion of the duodenum with which it did not show a clear plan of cleavage, measuring about $11.4 \times 6.2 \mathrm{~cm}$. The pancreas presented small dimensions, small scattered calcifications in the parenchyma. Dilation and tortuosity of the main pancreatic duct measuring $0.8 \mathrm{~cm}$.

Contrast abdominal tomography was performed, showing pancreas with signs suggestive of chronic pancreatitis and pseudocyst, as well as bulky expansive formation on the wall of the duodenum compatible with hematoma (Figures 1). In digestive endoscopy, the mucosa was located in the posterior wall of the right-pyloric cavity to the edge of the second duodenal portion suggestive of extrinsic compression (Figure 2).

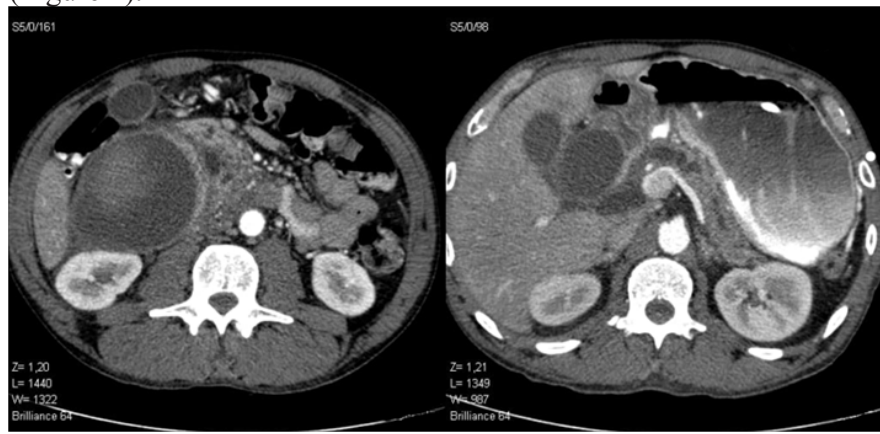

Figure I Tomography of the abdomen and pelvis with contrast showing pancreas with small dilatation and tortuosity of the main pancreatic duct and cystic formation at the head of the pancreas of about $3.5 \mathrm{~cm}$. Expansive formation in the lateral wall of the first and second duodenal portions, extending caudally to the right iliac fossa of about $16 \times 10 \times 8 \mathrm{~cm}(650 \mathrm{ml})$. The lesion was spontaneously hyper attenuating suggesting hematoma with recent bleeding.

In front of the findings, conservative treatment and monitoring were chosen, after two days of hospital stay, patient presented improvement of abdominal pain and good diet acceptance. On the 4thday, maintaining good general condition and hemodynamic stability, we opted for discharge without patient follow-up with early return. After 12days of discharge, the patient returned to present post-prandial discharge sensation, nausea and multiple episodes of vomiting with food debris and return of the ethyl habit.

Patient was again hospitalized, presenting increased levels of serumamylase $(518 \mathrm{U} / \mathrm{L})$ and hemoglobin of $10.2 \mathrm{~g} / \mathrm{dL}$. Considering the impossibility of feeding, a new high endoscopy was requested, which showed an increase in duodenal bulging that prevented the progression of the device. Naso enteral catheter was passed with the aid of guide wire. Until the 10th day of hospitalization, he had been presenting progressive clinical improvement, reduction of amylase 
levels. On the 11th day of hospitalization, he presented hemodynamic instability and died due to rupture of the duodenal hematoma not responding to cardiopulmonary resuscitation.

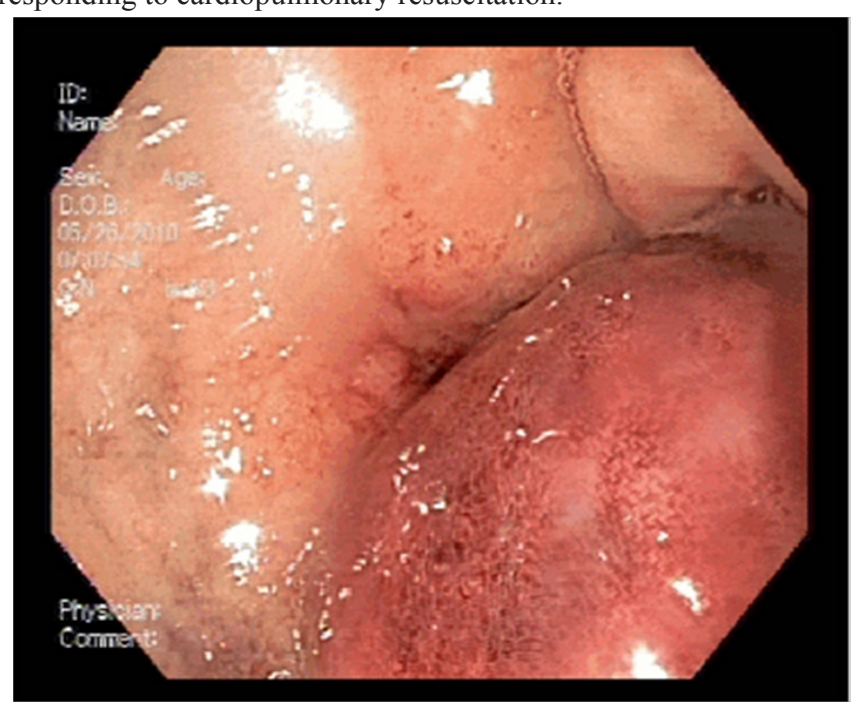

Figure 2 Abutment of the mucosa of these condportion of the duodenum.

\section{Discussion}

Chronic pancreatitis is a disease marked by the chronic inflammatory state of the pancreas with moments of exacerbation, which evolves with irreversible loss of endocrine and exocrine function. It presents an incidence of 4.4-11.9 cases per 100,000 inhabitants, with the male sex being 1.5-3times more affected. ${ }^{5}$ There are two hypotheses that try to explain the correlation of pancreatitis with HID formation, the first one defends the presence of ectopic pancreatic tissue located in the duodenum wall, which can evolve within flammation, necrosis and hematoma formation. The second hypothesis states that the increase of pancreatic enzymes causes lesion of vessels of the anteroposterior pancreato duodenal arcade. ${ }^{6}$

In a literature review performed by Oliveira et al., ${ }^{7}$ males were the most affected $(85.7 \%)$, ranging in age from 27years to 71 years (mean 53years). The alcoholic etiology of pancreatitis was the most common cause. The most common clinical condition is abdominal pain in the epigastric region, associated with episodes of recurrent vomiting, due to duodenal obstruction. . $, 3,8-13$ Obstructive symptoms usually resolve within $10-15$ days. ${ }^{14}$ Conservative treatment is often performed, consisting of naso gastric tube, antibiotic therapy, volume support and parenteral nutrition. ${ }^{7,12,15,16}$ When choosing conservative treatment, the patient should be monitored in order to diagnose possible complications, such as perforation and intestinal ischemia. ${ }^{12}$ Surgical treatment is classified when the patient does not respond to conservative treatment, evolving with worsening of symptoms, increased hematoma, perforation or intestinal ischemia, and hematimetricl levels. ${ }^{7,12}$

A surgical may be less invasive through endoscopic drainage of the hematoma. ${ }^{2,7,10}$ More aggressive surgeries such as and Whipple and Frey, in cases of pancreatitis due to oncologic suspicion or duodenal inviability. ${ }^{11,12,17}$

Because it is a rare condition, the treatment is controversial, in our report the patient presented improvement of the clinical and laboratory conditions with conservative treatment, but it evolved with rupture of the duodenum and death.

\section{Conclusion}

Duodenal intramural hematoma is a rare complication of pancreatitis few reported in the literature. The treatment is controversial, it should take in to consideration the clinical condition of the patient and the degree of duodenal involvement.

\section{Acknowledgments}

None.

\section{Conflicts of interest}

The author(s) declared no potential conflicts of interest with respect to the research, authorship, and/or publication of this article.

\section{References}

1. J Mc Lauchlan. Fatal false aneurysmal tumour occupying nearly the whole of the duodenum. Lancet. 1838;30:203-205.

2. Lee JY, Chung JS, Kim TH. Successful Endoscopic Decompression for Intramural Duodenal Hematoma with Gastric Outlet Obstruction Complicating Acute Pancreatitis. Clin Endosc. 2012;45(3):202-204.

3. Eurboonyanun C, Somsap K, Ruangwannasak S, et al. Spontaneous Intramural Duodenal Hematoma: Pancreatitis, Obstructive Jaundice, and Upper Intestinal Obstruction. Case Rep Surg. 2016;2016:1-4.

4. Veloso N, Amaro P, Ferreira M, et al. Acute pancreatitis associated with a nontraumatic, intramural duodenal hematoma. Endoscopy. 2013;45:E51-E52.

5. Conwell DL, Lee LS, Yadav D, et al. American Pancreatic Association Practice Guidelines in Chronic Pancreatitis. Pancreas. 2014;43(8):1143-1162.

6. van Spreeuwel JP, van Gorp LHM, Bast TJ, et al. Intramural Hematoma of the Duodenum in a Patient with Chronic Pancreatitis. Endoscopy. 1981;13(6):246-248.

7. Oliveira JHB de, Esper RS, Ocariz RC, et al. Intramural duodenal hematoma secondary to pancreatitis: case report and review of the literature. Sao Paulo Med J. 2017.

8. Bodnár Z, Várvölgyi C, Tóth J, et al. Intramural duodenal hematoma complicating acute necrotizing pancreatitis. Gastrointest Endosc. 2000;52(6):791-793.

9. Leundji H, Cuingnet P, Simon M, et al. Duodenal hematoma associated with thrombopenia in chronic alcoholic pancreatitis. Gastroenterol Clin Biol. 2002;26:185-186.

10. Fukunaga N, Ishikawa M, Yamamura Y, et al. Spontaneous intramural duodenal hematoma complicating acute pancreatitis. Surgery. 2011;149:143-144.

11. Neuzillet C, Facchiano E, Palazzo L, et al. Intramural duodenal hematoma as a complication of paraduodenal pancreatitis. Clin Res Hepatol Gastroenterol. 2011;35(2):140-142.

12. Ma JK, Ng KK, Poon RT, et al. Pancreatic-induced Intramural Duodenal Haematoma. Asian J Surg. 2008;31(2):83-86.

13. Kitadani J, Yamade N, Nakai H, et al. Intramural duodenal haematoma caused by pancreatic fistula due to exacerbation of chronic pancreatitis. BMJ Case Rep. 2018:bcr-2017-224058.

14. Simi S, Anoop TM, George KC. Spontaneous intramural duodenal hematoma-a rare cause of upper gastrointestinal obstruction. $\mathrm{Am} \mathrm{J}$ Emerg Med. 2010;28:642.e1-642.e2.

15. Dugernier TL, Breuskin FM. Duodenal Air Dissection Secondary to Intramural Hematoma in Necrotizing Pancreatitis. Endoscopy. 2002;34(12):1024-1024. 
16. Khurana T, Shah A, Ali I, et al. Intramural Duodenal Hematoma with Acute Pancreatitis in a Patient with an Overt Pancreatic Malignancy. ACG Case Reports J. 2014;1(4):209-211.
17. Molina-Barea R, Pérez-Cabrera B, Hernández-García MD, et al Abdomen agudo por hematoma duodenal intramural complicado. Presentación de un caso y revisión en la literatura. Cir Cir. 2015;83:146-150. 